Promoting Open Science Practices: What Can (Should) Graduate Programs Do?

\begin{abstract}
The training that applied linguists receive in graduate school can have a long-lasting impact on their career and research practices. Collectively, the field must therefore consider how to best educate upcoming generations of researchers to see Open Science practices as the standard rather than as the exception. In this chapter, we describe what we are eager to see in graduate programs that not only advocate for Open Science, but also proactively equip students with knowledge and skill sets to embrace the future research environment. We propose a more systematic integration of Open Science into the training of research methods, both within individual courses and across the program at large. We conclude by pointing readers to a short survey quiz for graduate students and program directors to self-assess their readiness to welcome an Open Science future in applied linguistics.
\end{abstract}




\section{Promoting Open Science Practices: What Can (Should) Graduate Programs Do?}

"And variation in (research) practice is partly heritable, in the sense that apprentices acquire research habits and statistical procedures from mentors and peers." (Smaldino \& McElreath, 2016, p. 2)

In recent years, there have been numerous calls for applied linguists to embrace Open Science (OS) practices as a means to enhance transparency and therefore study quality (e.g., (Godfroid \& Hui, 2020; Loewen \& Godfroid, 2020; Marsden \& Plonsky, 2018). The field's response has been generally positive. For example, at least six journals in the field award Open Science Badges to authors who follow these practices (see https://www.cos.io/our$\underline{\text { services/badges for a full list). Fifty-four journals, as well as national and international }}$ teaching and research associations, such as the American Association of Applied Linguistics and the British Association for Applied Linguistics, furthermore support materials sharing through IRIS, a digital repository of instruments and materials for research into second languages (see https://www.iris-database.org/ for a full list). The journals Language Learning and Bilingualism: Language and Cognition, among others, offer registered reports as an article type in addition to the regular, conventional submission route. Studies in Second Language Acquisition, Language Teaching, and Applied Psycholinguistics have a dedicated section for replication research. Furthermore, Language Learning now requires authors to submit their materials for review along with the manuscript (Marsden et al., 2019). Applied Psycholinguistics has also made it mandatory for authors to share their experimental 
materials as well as analysis code and data beginning July 2021 and January 2022, respectively ${ }^{1}$.

From this list of examples, it is clear that the field is rapidly evolving its attitudes and expectations about OS. Practices which are considered "nice, but optional" today will very likely become expected standard protocol over the next decade. When this is the case, any awareness of the trend should elicit a long, hard look in the mirror. Individual researchers must ask themselves if they are ready and willing to accept and promote such changes. Graduate programs must also acknowledge their obligation in developing and supporting the establishment of OS practices in applied linguistics through research training. The key question for self-reflection, therefore, is not just, “am I ready to embrace OS practices?", but “is today's field equipped to train future generations of researchers in OS practices?” More specifically, are our students prepared to embrace OS as the future norm in research? What should a graduate program that equips students for OS look like? In this chapter, we offer a vision of how programs should comprehensively build OS practices into their graduation milestones. We next discuss ways in which OS can be incorporated into methods training and individual, theoretically oriented content courses. Finally, we conclude by pointing readers to a self-assessment tool, which they can use to understand their current standing.

\section{Integrating OS at the Program Level: A Comprehensive Incentive Structure}

\footnotetext{
${ }^{1}$ While we are eager to recognize the efforts of different parties in promoting Open Science practices, we are unable to provide an exhaustive list. We apologize for missing any important endeavor in this list of examples.
} 
For many graduate educators, the thought of adding OS training into an already-packed graduate curriculum can seem like a tall order. However, many pre-existing program structures can be easily tweaked to embrace OS practices. In this section, we discuss ways in which programs can integrate OS into their program requirements and curricula. By highlighting and incentivizing OS practices from the outset, graduate programs will equip graduate students to be aware of and able to practice OS from the outset.

\section{Qualifying Research Papers and Pre-registration}

In many graduate programs, students write research papers as (part of) their qualifying exams. We envision that programs could adopt the practice of pre-registration as a formal benchmark in this process. When researchers formally pre-register a study's protocol, they commit to not making any ex post facto changes in the methodology or analyses. Although that is not to say they cannot engage in exploratory investigation after data collection (they must make the exploration explicit, rather than presenting the effort as confirmatory), preregistration means that researchers must critically assess and optimize the research plan $a$ prior. Indeed, such deliberation serves as an excellent training opportunity for graduate students. By treating pre-registration as a project "checkpoint," students can focus on refining their research design and identifying potential limitations before beginning their study. Incorporating pre-registration into qualifying research papers does not necessarily mean that students must formally pre-register with a journal (i.e., submit a registered report); rather, programs could replicate the pre-registration process internally. For example, instead of submitting a completed manuscript to their readers (examiners), students would first submit study protocols for a stage-one review. Once the readers have approved designs, materials, and analysis plans, students would officially pre-register their studies on a platform, such as Open Science Framework (OSF; www.osf.io) or on a departmental drive, and carry out the 
study according to the plan. When the study is complete, a full manuscript can then be submitted to the readers for a stage-two review, fulfilling the research paper requirements. When the student chooses to pre-register their study on a public platform (e.g., OSF), they can indicate that they have pre-registered their study when they publish their findings. The pre-registration qualifies them for a pre-registration badge, one of the OS badges that journals award to researchers to recognize their effort and commitment to OS practices. While there is little information available on how OS practices are currently weighed in appointments (Schönbrodt, 2019), we have increasingly seen them listed in job advertisements in such neighboring fields as psychology. Thus, for students on the job market, an OS badge associated with a publication demonstrates not only their ability to publish research, but also their commitment - ahead of the curve - to Open Science. In the end, the ultimate choice of pre-registration platform is not as important as the fact that the process of pre-registration will scaffold graduate learning. Breaking down the research process into its component parts is pedagogically more effective than expecting students to master all the skills involved in the entire research cycle in one go. Rather than expecting students to submit a full paper of publishable quality from the get-go, students will need to focus on and perfect the foundational stages of their research proposal before they can move on to data collection.

In addition to its pedagogical benefits, we argue that incorporating pre-registration as a program benchmark is ultimately more resource-effective. Students typically have limited time and funding to carry out their research within the confines of a fixed term graduate program. By integrating pre-registration into the qualifying research paper process, examiners can help students identify potential methodological flaws prior to data collection. Even the most successful senior scholars can relate to the frustrating experience of finding out major, unacceptable flaws in the study design at a later stage of research (e.g., publishing). For scholars-in-training who are still honing their research skills, such flaws are understandably 
more common. Program-initiated pre-registration could prevent the depletion of a graduate student's already-limited resources (time, funds) on qualifying papers which are ultimately unviable for actual publication. We envision that the student's revision of the methodology, based on examiner feedback, would become part of the formal qualifying exam evaluation process. With help of focused feedback early on in the research process, adopting the practice of pre-registration may also improve the overall standard of such qualifying papers, making eventual publication all more likely.

\section{Methods Training and Open Data}

In applied linguistics, the use of published data for a new study is still uncommon, but not unprecedented. Corpus researchers, for example, use learner corpora as observational data to address new questions (e.g., Tracy-Ventura \& Huensch, 2018). Openly published online measures also provide opportunities for renewed analyses (e.g., Elgort et al., 2018; Hui, 2020). Graduate programs should work to intentionally incorporate open data into their methods training. From a pedagogical perspective, both qualitative and quantitative methodology courses can utilize published data to teach field-relevant analyses. By studying the accompanying codebooks and software code, graduate students can learn how to program in software packages (e.g., R), how to perform specific statistical analyses in the programming language, and how to select the information from the software output for reporting. The data processing pipeline of a successful publication serves as a model flow for students to manage, process, analyze, and share their own data and software code. Taking one step further, students can also deliberate on how existing data might address novel research questions as they engage in open data sets. Since published data have already passed peer reviews for the original study, graduate students can take advantage of the good data quality (i.e., obtained from a rigorous research design) to pursue new research questions and 
analyses. In other words, exposure to open data sets can help students familiarize themselves with how to access and utilize the very many openly available resources, potentially saving them from the time and money needed for a fresh round of data collection.

In addition, after seeing how other researchers share their data and software code, graduate students can also benefit from making their own data and code open. Not only can they list their data publication alongside their article in their resume, but furthermore they may obtain more citations for both (Leitner et al., 2016; Piwowar et al., 2007; Piwowar \& Vision, 2013). Per the author guidelines of Applied Linguistics, for example, researchers using a publicly available data set must fully reference it. Although data citation is not yet common in our field, we predict that data publications will be valued on the job market and future tenure promotion as OS becomes more widely recognized and valued. However, it must be acknowledged that the open sharing of data and analyses is a time-consuming endeavor. As noted previously, graduate students are operating under a constricted time frame in which they must prove mastery in multiple domains. Taking on the additional burden of preparing the files, identifying a suitable repository, and curating the entry might be seen as a relatively low priority. From our own experience, however, making our data open encourages us to add an extra layer of care in all steps of the research process. Not only do we carefully document changes and updates to the analyses in a project log, but we furthermore annotate our data and analyses with external readers in mind. Details such as these almost enhance the quality and rigor of one's work.

Finally, the incorporation of open data into a graduate curriculum can support other areas of training such as research ethics and data management. To successfully publish data, authors must familiarize themselves with the logistical demands (i.e., curation and management of data) and ethical requirements (e.g., anonymity). Graduate students can evaluate the extent to which their own data meet these sharing prerequisites, either with their 
advisor or with their Institutional Review Board. Programs could also require that graduate students submit a formal data-sharing plan as a part of their qualifying research paper examination. Indeed, many funders now do require such as a plan in grant applications. Therefore, this intramural practice will put the student in a better position when they apply for grants for their dissertation research and indeed for their future work as an independent researcher. In sum, by requiring students to think about data sharing for their qualifying research papers, programs can incorporate multiple curricular domains (research ethics, data management, open science practices) into a final product which will benefit their students.

\section{Other ways to incentivize OS at the Program Level}

We must acknowledge that the hands of the faculty and program directors are often tied when there are competing priorities. Due to various reasons, a program may not be ready to wholeheartedly integrate OS into their graduate requirements. But, these constraints should not stop graduate programs from encouraging students to adopt OS. Here, we list several policies that can be adapted as a catalyst for discussion and implementation:

1. Research Funding and Fellowship Applications: in applications for research funding or fellowships, programs can include a section for OS practices. Programs can stipulate that the adoption of OS practices is one of the criteria by which applications will be evaluated.

2. Replication for Research Papers: Programs can encourage students to replicate an existing study for their early qualifying research papers (or for MA theses). In doing so, the focus is thus placed on methodological rigor over the pressure to execute original ideas.

3. Host OS workshops: programs host OS workshops and how-to sessions for students. These sessions can be held jointly with other units across the university 
and/or with other experts in our field. Students will have the opportunity to see how OS is practiced in real life. Programs can also invite other OS-oriented personnel on campus (such as librarians, statistical analysts, and IRB staff) to give guest lectures.

4. Create an OS reading group: around the globe, many programs across disciplines have started their OS-themed reading groups or journal clubs, either on their own (e.g., the Open Science Reading Group at Standford Medicine [https://laneguides.stanford.edu/open-science-reading]) or through initiatives such as ReproducibiliTea (https://reproducibilitea.org/). In the spirit of OS, groups often publicly share their reading lists and discussion questions so that others can freely borrow and adapt accordingly (e.g., see https://osf.io/3ed8x/ for a starter pack shared by the ReproducibiliTea initiative).

5. Preparing for the job market: most graduate programs host workshops to help students prepare for the academic job market as they approach graduation. During these workshops, programs can highlight examples of how students might incorporate their own practice of OS into resumes as well as cover letters. Programs can also inform students that their OS practices will be included in letters of reference.

\section{Integrating OS at the Course Level: A tiered approach to OS methods training}

Not all who support the integration of OS methods into graduate training will find themselves in a program that is willing and able to fully adopt OS. In this section, we discuss ways in which graduate educators could integrate OS practices into their own individual courses. 
For many OS advocates, the topics around OS could easily fill a semester-long course of content to teach. For example, a course can start with introducing students to what it means for applied linguistics to be a scientific discipline, the history of the OS movement as driven by the issues with replicability and questionable research practices in our own and neighboring fields. Against this background, students can further dive into OS practices such as pre-registration, open data and materials, not only gaining understanding at a theoretical level, but also knowing the nitty-gritty of the how-tos. If readers are in a position to offer such a course, we refer them to an Open Science Framework site where OS enthusiasts who actually do teach stand-alone OS courses share their course syllabi publicly (https://osf.io/vkhbt). The Framework for Open and Reproducible Research Training (FORRT; https://forrt.org/) has also identified seven clusters of knowledge and skills in their Open and Reproducible Science taxonomy. The seven clusters are: 1) reproducibility and replicability, 2) conceptual and statistical knowledge, 3) reproducible analyses, preregistration, 4) FAIR data and materials, replication research, and academic life and culture. Under each cluster (e.g., conceptual and statistical knowledge), there are subcategories of topics (e.g., logic of null-hypothesis significance testing and its benefits and limitations) that one can cover in a course on OS. Importantly, the site provides suggested reading lists for course instructors to consider including in their own syllabus. These example resources represent the tremendous and generous effort of the OS community to help each other promote training for future scientists across disciplines, which means that applied linguists who train the next generations of researchers do not necessarily need to reinvent the wheel in their course design. On the contrary, they can (and should) take advantage of the lessons learned in other neighboring disciplines which already have a more structured OS curriculum in place. 
While we believe that such a class on OS should be offered for all applied linguists in training (and indeed all aspiring scientists across disciplines), we also acknowledge that a stand-alone course approach may not be the most feasible option in the current state of graduate programs in applied linguistics for various reasons. For example, some topics might be new to many in our field, including the instructor themselves; hence it takes time for one to develop, adapt, and tailor course materials for an applied linguistics audience. There will also always be competing priorities as well as resources (e.g., teaching load) and curricular (core $v s$. elective courses) constraints which determine course offerings. Therefore, many graduate programs should at this point primarily consider integrating the OS content into the existing curriculum. We put much emphasis on integration because we also firmly believe that blindly expanding a graduate curriculum will result in compromising the quality of teaching and learning. Realistically, one cannot pack more into an already full suitcase (Well, many travelers hold a belief that a suitcase is never full). When integration is a primary goal, we would also like to further point out the different levels of integration that can be considered. In Table 1, we present a visualization of these potential levels. We order these levels according to the additional commitment required of/for the instructor and the program. For example, when a program is to offer a stand-alone course on OS (top level 1), adequate resources (time and funds) need to be made available for the development and implementation of the course. Despite the comprehensiveness of the resulting course, considerable deliberation and careful planning are needed, meaning that this level of integration is not ready for immediate implementations. In contrast, we argue that integration at the lowest level is something that almost all instructors can do for the next semester. Commitment is minimal but such integration can already provide students with exposure to some OS content. The downside for this level of integration might be that the teaching is less structured and hence some degree of coordination is needed at the program level. As 
mentioned, the lower levels appear to be more realistic for many applied linguistics programs; therefore we focus our discussion on these lower levels. Given the wealth of information available in the OS community, we reserve the space for exemplifying how OS topics can be integrated into existing graduate training, instead of attempting an exhaustive list of OS content.

Table 1

A Tiered Approach to Content and Open Science Integration

\begin{tabular}{|c|c|c|}
\hline Levels & Descriptions & Examples \\
\hline $\begin{array}{l}\text { Level } 1 \text { - Stand-alone OS } \\
\text { course }\end{array}$ & $\begin{array}{l}\text { Instructor offers a stand- } \\
\text { alone course on OS }\end{array}$ & $\begin{array}{l}\text { Instructor offers Conducting } \\
\text { Open Research (potentially } \\
\text { collaboratively with other } \\
\text { social sciences programs) }\end{array}$ \\
\hline $\begin{array}{l}\text { Level } 2 \text { - Full Content-OS } \\
\text { Integration }\end{array}$ & $\begin{array}{l}\text { Instructor teaches OS skills } \\
\text { along with existing content } \\
\text { in a parallel manner }\end{array}$ & $\begin{array}{l}\text { Instructor covers coding in } \mathrm{R} \\
\text { along with statistical } \\
\text { concepts. }\end{array}$ \\
\hline $\begin{array}{l}\text { Level } 3 \text { - Partial Content-OS } \\
\text { Integration }\end{array}$ & $\begin{array}{l}\text { Instructor dedicates specific } \\
\text { class time and/or assignment } \\
\text { to cover OS topics. }\end{array}$ & $\begin{array}{l}\text { Instructor of general } \\
\text { research methods devote the } \\
\text { first two weeks to topics } \\
\text { such as biases in research, } \\
\text { reproducibility, among } \\
\text { others. } \\
\text { Instructors could ask for } \\
\text { proposals of replication } \\
\text { studies (rather than an } \\
\text { original study proposal) for } \\
\text { the end of semester project }\end{array}$ \\
\hline $\begin{array}{l}\text { Level } 4 \text { - Basic, Minimal } \\
\text { Content-OS Integration }\end{array}$ & $\begin{array}{l}\text { Individual instructor } \\
\text { considers OS when selecting } \\
\text { teaching materials in order } \\
\text { to provide exposure and } \\
\text { discussion opportunities as } \\
\text { the theoretical content is } \\
\text { taught. }\end{array}$ & $\begin{array}{l}\text { Instructor includes registered } \\
\text { (replication) reports } \\
\text { published in the content } \\
\text { domain in their reading list. } \\
\text { Instructor demonstrates an } \\
\text { experiment task using open } \\
\text { materials to show how a } \\
\text { theoretical construct is } \\
\text { measured and } \\
\text { operationalized. }\end{array}$ \\
\hline
\end{tabular}


We start with discussing how an instructor can incorporate OS in their own content courses. Since graduate-level courses often adopt published articles as the main source of readings, we argue that there is much room to integrate OS in the selection of readings. For example, articles with publicly available materials are prime candidates for content courses because instructors can easily incorporate these materials directly into the classroom to illustrate new constructs and tools. Exposure to open materials can be an eye-opening experience because graduate students (and senior researchers alike) must have experienced the frustration of trying to make sense of an experimental protocol from space-constrained prose alone. Being able to inspect the actual, full set of materials can facilitate a critical assessment of the study's methodology as well as the validity of the results and conclusion. In a long run, students will be able to design their own materials for new purposes. At the same time, we acknowledge that developing reliable and valid materials is not an easy task after all. It requires sound understanding of the theoretical construct in addition to challenging and often highly technical issues of psychometric properties. When graduate students have exposure to open materials, they are aware of the many repositories that they can visit to locate study materials for their own work. In general terms, use of open materials puts researchers in a better position to compare their own work with the existing literature. Further, use of open materials essentially means that they take advantage of other researchers' previous experience. The efforts saved by not having to "reinvent the wheel" can then be put towards other tasks. Imagine where the field could be if the time saved was compounded across the careers of an entire generation of researchers. This is not to say open materials are a panacea, nor that researchers should blindly adopt open materials across contexts. Researchers are still responsible for assessing, for example, the reliability of the instruments in their sample. Taken together, one key is therefore to teach students to make 
searching for open materials a default when designing their study. With critical assessment of appropriateness, they decide what adaptation is necessary. In cases where new materials need to be developed, they should also (know how to and where to) share them.

When instructors feel more ready to discuss OS with students, they can indeed devote a certain amount of time to OS content. Potentially, this level of integration is most appropriate to introduction to research methods at the graduate level. If basic methods training is assumed upon admission, this OS content can be placed in introductory graduate seminars. In either case, the content focus of a given (number of) week(s) can be on OS topics such as biases in research, reproducibility, preregistration, among others. In discussing preregistration, for example, students need articulate/know to the distinction between exploratory and confirmatory research and how formally pre-registering a study's protocol can guard against any ex post facto changes in the methodology or analyses and questionable research practices such as $p$-hacking (e.g., attempting various configurations of analyses only for statistical significance) and HARK-ing (i.e., Hypothesizing After Results are Known). They also need to know how they can do it themselves on platforms such as Open Science Framework (www.osf.io) and what content should be included in a study protocol (see https://osf.io/zab38/ for a list of useful templates). As an alternative route, students should be aware that some journals in our field are accepting registered report submissions and the difference in the review process between this and the conventional routes. That is, initial peer review takes place before data collection. At this stage, the peer review focuses on "whether the research question(s) is/are justified and valuable and whether the proposed design, methods, and analyses are sound” (Marsden et al., 2018, p. 313). If passed, the journal will issue an in-principle acceptance (IPA) to the authors. Then, the authors collect and analyze the data strictly according to their proposal. After the results and discussion have been written, the manuscript is submitted for a stage-two peer review. Importantly, "reviewers [at 
this second stage] cannot recommend rejecting a manuscript on the basis of the justification or methods that were accepted at stage one" (Marsden et al. 2018 p. 314). In other words, if the study is conducted according to the registered protocol, an IPA is an almost guaranteed acceptance. Since discussion on a topic such as this one will benefit from having both theoretical (e.g., why preregister?) and practical (e.g., what is the review process like?) angles, we believe some dedicated time in the syllabus is more appropriate as it can ensure a deeper understanding and immersed experience in the how-tos.

Finally, there are skill sets that OS calls for that are best taught in tandem with existing content. Coding skills in a programming language such as $\mathrm{R}$ is a point in case. As pointed out by Mizumoto and Plonsky (2016) the programming $\mathrm{R}$ is a prime candidate for a common language in the field, partly because it is easy to reproduce data analyses in R. In the light of OS, the ability to repeat an analysis and arrive at the same conclusion is a basic, fundamental level of reproducibility. Publishing R code will make it possible for peers to check (and potentially correct) published findings (Obels et al., 2020). More to this is the reproducibility of the data preparation pipeline prior to actual model fitting. Indeed, a lot of researchers' degrees of freedom lie in the data preparation process (e.g., ad-hoc removal of outliers). To be embrace research transparency, researchers should document and share the entire data processing pipeline. On this account, then, using $\mathrm{R}$ to conduct data pre-processing as well as data analysis is an essential skill to learn for graduate students even though there are other possible ways to achieve the same goals. Learning $\mathrm{R}$ is not very different from learning a language, which many of the readers are an expert. Specifically, the language is best learned and taught in context. Integrating such coding skills into a quantitative methods class will provide students with hand-ons experience. What instructors can do is to break down each session devoted to a given statistical topic (e.g., correlations) into a theory and a practical part, which believe is also a common practice regardless of software packages. In 
the first part, the statistical concepts are introduced and discussed; in the second, emphasis can be put on using $\mathrm{R}$ to execute the relevant analysis. At this time, we acknowledge that, as with all other OS content, coding might be new to the instructor themselves, and so one may or may not feel comfortable teaching it. We would like to offer two potential solutions: first, the instructor might use a statistics textbook that incorporates coding in R (e.g., Loerts et al., 2020). Alternatively, some more senior $\mathrm{PhD}$ students might already have learned $\mathrm{R}$ on their own because their work needs it. If that is the case, these students can become a teaching assistant in a quantitative methods class where they teach their junior peers how to code in R. This experience will also help the senior student build a more comprehensive teaching profile when they are on the job market. Finally, we wanted to acknowledge learning (and teaching) statistics and coding together is an easy task, to say the least. Instructors and students can further explore resources available online (e.g., PsyTeachR [https://psyteachr.github.io/]) and/or on campus (e.g., meetup groups, student groups for data science that hold regular workshops). As with all other teaching materials, they can be recycled in further years.

In sum, we discussed how course instructors can integrate OS content in their existing graduate curriculum. The different levels of integration require various degrees of commitment. With some program-level coordination and individual instructor's planning and thoughts, students will benefit hugely from a more structured curriculum that is OS supportive.

\section{Concluding Remarks}

Early in the chapter, we argued that graduate training can have a long-lasting impact on researchers' practice. We also included a quote from Smaldino and McElreath (2016) describing how (both good and bad) research practices can be passed along to the next generations through mentorship. We cannot stress the role of graduate programs more in 
promoting OS practices. Therefore, we humbly call for a concerted effort to promote OS through the training of graduate students, the next generations of researchers, in OS practices. Graduate directors and faculties should start evaluating how well they are preparing their students for the future research environment and reflect on what values, knowledge, and skills are essential for students to excel in academia. To do that we point readers to a selfassessment tool from FORRT (https://forrt.org/self-assessment/) which graduate students and program directors can use to take the very first step towards change. Not only can this assessment shed light on our current standing, it also represents a needs analysis to identify areas that a program has already been doing well and those that need further strengthening. Although change does not happen overnight, the different levels of integration of OS content that a graduate curriculum can achieve provides a menu of options for graduate programs to move forward. Through allowing students to engage in OS, students will adopt an OS mindset that they can take with them throughout their career. Graduate educators are in a very special position because they can choose the good genes to pass along their academic bloodline, planting the seeds of an OS future in applied linguistics. 


\section{References}

Elgort, I., Brysbaert, M., Stevens, M., \& Van Assche, E. (2018). Contextual word learning during reading in a second language: An eye-movement study. Studies in Second Language Acquisition, 40(2), 341-366. https://doi.org/10.1017/S0272263117000109

Godfroid, A., \& Hui, B. (2020). Five common pitfalls in eye-tracking research. Second Language Research, 36(3), 277-305. https://doi.org/10.1177/0267658320921218

Hui, B. (2020). Processing variability in intentional and incidental word learning: An extension of Solovyeva and Dekeyser (2018). Studies in Second Language Acquisition, 42(2), 327-357. https://doi.org/10.1017/S0272263119000603

Leitner, F., Bielza, C., Hill, S. L., \& Larrañaga, P. (2016). Data publications correlate with citation impact. Frontiers in Neuroscience, 10. https://doi.org/10.3389/fnins.2016.00419

Loerts, H., Lowie, W., \& Seton, B. (2020). Essential statistics for applied linguistics using $R$ or JASP (2nd ed.). MacMillan.

Loewen, S., \& Godfroid, A. (2020). Advancing quantitative research methods. In H. Rose \& J. McKinley (Eds.), The Routledge handbook of research methods in applied linguistics (pp. 98-107). Taylor and Francis.

Marsden, E., Crossley, S., Ellis, N., Kormos, J., Morgan-Short, K., \& Thierry, G. (2019). Inclusion of research materials when submitting an article to Language Learning. Language Learning, 69(4), 795-801. https://doi.org/10.1111/lang.12378

Marsden, E., Morgan-Short, K., Trofimovich, P., \& Ellis, N. C. (2018). Introducing Registered Reports at Language Learning: Promoting Transparency, Replication, and a Synthetic Ethic in the Language Sciences: Introducing Registered Reports. Language Learning, 68(2), 309-320. https://doi.org/10.1111/lang.12284 
Marsden, E., \& Plonsky, L. (2018). Data, open science, and methodological reform in second language acquisition research. In A. Gudmestad \& A. Edmonds (Eds.), Critical reflections on data in second language acquisition (pp. 219-228). John Benjamins.

Mizumoto, A., \& Plonsky, L. (2016). R as a lingua franca: Advantages of using R for quantitative research in applied linguistics. Applied Linguistics, 37(2), 284-291. https://doi.org/10.1093/applin/amv025

Obels, P., Lakens, D., Coles, N. A., Gottfried, J., \& Green, S. A. (2020). Analysis of open data and computational reproducibility in registered reports in psychology. Methods and Practices in Psychological Science, 3(2), 229-237. https://doi.org/10.1177/2515245920918872

Piwowar, H. A., Day, R. S., \& Fridsma, D. B. (2007). Sharing detailed research data is associated with increased citation rate. PLoS ONE, 2(3), e308. https://doi.org/10.1371/journal.pone.0000308

Piwowar, H. A., \& Vision, T. J. (2013). Data reuse and the open data citation advantage. PeerJ, 1, e175. https://doi.org/10.7717/peerj.175

Schönbrodt, F. (2019). Training students for the Open Science future. Nature Human Behaviour, 3(10), 1031-1031. https://doi.org/10.1038/s41562-019-0726-z

Smaldino, P. E., \& McElreath, R. (2016). The natural selection of bad science. Royal Society Open Science, 3(9), 160384. https://doi.org/10.1098/rsos.160384

Tracy-Ventura, N., \& Huensch, A. (2018). The potential of longitudinal learner corpora in SLA research. In A. Gudmestad \& A. Edmonds (Eds.), Critical reflections on data in second language acquisition (pp. 219-228). John Benjamins. 